\title{
FACTORES DETERMINANTES DE EMBARAZO POSTERIOR A REANASTOMOSIS TUBÁRICA REALIZADAS EN EL HOSPITAL REGIONAL TEODORO MALDONADO CARBO; PERIODO ENERO 2010 A JUNIO 2015
}

\author{
DETERMINING FACTORS OF PREGNANCY POST TUBARIC REANASTOMOSIS PERFORMED IN THE \\ REGIONAL HOSPITAL TEODORO MALDONADO CARBO BETWEEN JANUARY 2010 AND JUNE 2015
}

DETERMINANDO FATORES DE GRAVIDEZ APÓS A REANASTOMOSE TUBARICA REALIZADA NO HOSPITAL TEODORO REGIONAL MALDONADO CARBO ENTRE JANEIRO 2010 E JUNHO DE 2015

\author{
ALEJANDRA MARÍA YU LEE MATEUS', MARÍA GRACIA SALAZAR DIK', DIEGO ANTONIO VÁSQUEZ CEDEÑO' \\ 1 Universidad Católica de Santiago de Guayaquil, Guayaquil, Ecuador
}

\begin{abstract}
Resumen
Introducción: la obstrucción tubárica constituye el 35\% de las causas de infertilidad, La ligadura de trompas de Falopio es el método anticonceptivo elegido por la mayoría de la población femenina fértil a nivel mundial. Sin embargo, del 1 al $5 \%$ deseará la reversión de la cirugía en los años subsecuentes. Objetivos: determinar los factores que favorecen el embarazo posterior a la reanastomosis tubárica. Método: estudio cohorte retrospectivo de historiales clínicos de pacientes esterilizadas, que posteriormente se realizaron la reanastomosis tubárica en el hospital Teodoro Maldonado Carbo, periodo enero 2010 a junio 2015 , obteniendo una muestra final de 51 pacientes. Resultados: se obtuvo un total de 28 gestas pertenecientes a 18 pacientes, de las cuales 5 fueron intrauterinos viables (17.9\%). En el 67.9\% de las gestas, la recanalización fue bilateral; en los embarazos uterinos viables se realizó anastomosis ístmico-ístmica. El promedio de tiempo transcurrido desde la recanalización hasta la primera gestación fue de $8.4 \pm 5.6$ meses. Conclusión: en el estudio la reanastomosis tubárica alcanzó una tasa de éxito clínicamente significativa, pero no garantiza la viabilidad de un embarazo. Cuando la reanastomosis no resulte favorable, se puede plantear la terapia de reproducción asistida para lograr la concepción.
\end{abstract}

PALABRAS CLAVE: trompas uterinas, esterilización tubaria, infertilidad, embarazo.

Abstract

Introduction: tubal obstruction constitutes $35 \%$ of the causes of infertility. Fallopian tube ligation is the contraceptive method chosen by the majority of the fertile female population worldwide. However, from 1 to $5 \%$ of patients will want the reversal of the procedure in subsequent years. Objectives: to determine the factors that favor pregnancy after tubal reanastomosis. Methods: this is a retrospective cohort study of clinical records of sterilized patients, who subsequently underwent tubal re-anastomosis at Teodoro Maldonado Carbo Hospital, from January 2010 to June 2015, obtaining a final sample of 51 patients. Results: a total of 28 gestations belonging to 18 patients were obtained, of which 5 were intrauterine viable (17.9\%). In $67.9 \%$ of the deeds, the recanalization was bilateral; In viable uterine pregnancies, isthmic-isthmic anastomosis was performed. The average time from recanalization to first pregnancy was $8.4 \pm 5.6$ months. Conclusion: in the study, tubal reanastomosis reached a clinically significant success rate, but it does not guarantee the viability of a pregnancy. When the reanastomosis is not favorable, assisted reproduction therapy can be considered to achieve conception. KEYWORDS: uterine tubes, tubal sterilization, infertility, pregnancy.

Resumo

Introdução: a obstrução tubária constitui 35\% das causas de infertilidade. A ligadura da trompa de Falópio é o método contraceptivo escolhido pela maioria da população feminina fértil em todo o mundo. No entanto, de 1 a $5 \%$ vão querer a reversão da cirurgia nos anos subseqüentes. Objetivos: determinar os fatores que favorecem a gravidez após a reanastomose tubária. Métodos: uma coorte retrospectivo de pacientes esterilizados registros médicos, depois anastomose tubária foram realizadas no Hospital Teodoro Maldonado Carbo, a partir de Janeiro de 2010 e Junho de 2015, a obtenção de uma amostra final de estudo 51 pacientes. Resultados: foram obtidas 28 gestações pertencentes a 18 pacientes, das quais 5 eram intrauterinas viáveis (17,9\%). Em 67,9\% dos atos, a recanalização foi bilateral; Em gestações uterinas viáveis, foi realizada anastomose istmo-ístmica. 0 tempo médio entre a recanalização e a primeira gestação foi de 8,4 $\pm 5,6$ meses. Conclusão: no estudo, a reanastomose tubária atingiu uma taxa de sucesso clinicamente significativa, mas não garante a viabilidade de uma gravidez. Quando a reanastomose não é favorável, a terapia de reprodução assistida pode ser considerada para alcançar a concepção.

PALAVRAS-CHAVE: tubas uterinas, esterilização tubária, infertilidade, gravidez. 


\section{INTRODUCCIÓN}

La reanastomosis tubárica (RAT) es un procedimiento quirúrgico en el que se unen dos porciones de la trompa de Falopio con el fin de restablecer la fertilidad de la mujer. Este método, también conocido como recanalización tubárica, puede ser empleado tanto por motivos de infertilidad como de reversión de esterilidad y se realiza uni o bilateralmente, dependiendo del caso., ${ }^{1,2}$

La infertilidad puede atribuirse a causas patológicas como la obstrucción tubárica que representa el 35\% de los casos y son la segunda causa de infertilidad más común después de la infertilidad de origen desconocido., ${ }^{1,2}$ Además, puede ser inducida mediante la esterilización quirúrgica conocida como ligadura de trompas de Falopio. Éste es el método anticonceptivo definitivo más común a nivel mundial. ${ }^{3,4}$ Se estima que alrededor de 138 millones de mujeres en edad reproductiva se encuentran esterilizadas, mientras que 100 millones más, optarán por este método en los próximos 20 años. ${ }^{3,4}$ Sin embargo, a pesar de un exhaustivo asesoramiento, se calcula que aproximadamente del $1 \%$ al $5 \%$ de las mujeres esterilizadas deciden revertir el procedimiento, e incluso, grandes estudios reportan un porcentaje de hasta $30 \%{ }^{2,5}$ Los motivos principales comprenden: edad temprana al momento de la esterilización, cambio de pareja, paridad de 2 o menos o la muerte de un hijo. $3,7,8$

El propósito de este estudio es determinar los factores que favorecen la concepción de un embarazo viable posterior a la reanastomosis tubárica realizadas entre enero de 2010 y junio de 2015 en el hospital regional Teodoro Maldonado Carbo de la ciudad de Guayaquil; y además, establecer los motivos que conllevan a la reversión de la esterilización tubárica, precisar el índice éxito y fracaso del procedimiento y proporcionar futuras recomendaciones para promover una planificación familiar confiable y definitiva.

\section{METODOLOGÍA}

Se realizó un estudio cohorte retrospectivo, que tiene como objetivo determinar los factores que favorecen el embarazo posterior a la reanastomosis tubárica. En este estudio se midió la probabilidad de embarazo según el tiempo transcurrido en unidad de meses desde la fecha de RAT hasta el embarazo de las pacientes.

Los criterios de inclusión fueron mujeres con antecedente de infertilidad de origen tubárico o esterilización tubárica quirúrgica e intervención quirúrgica de reanastomosis tubárica en el hospital regional Teodoro Maldonado Carbo de la ciudad de Guayaquil, desde enero de 2010 a junio de 2015. Se excluyeron a pacientes cuya reanastomosis tubárica fue realizada en otro centro hospitalario y pacientes que no continuaron con el control ginecológico y obstétrico postquirúrgico.

Las variables maternas que se midieron fueron: edad al momento de la esterilización (en años), edad al momen to del RAT (en años), motivo por el que deseaban concebir (deseo de procrear con la misma pareja, nueva pareja sin hijos, nueva pareja con hijos, hijo fallecido), antecedentes ginecobstétricos (partos, gestas, cesáreas, abortos), parámetros hormonales (perfil lipídico, glicemia e Índice de Resistencia a la Insulina(HOMA-IR) HOMA) y de Índice de Masa Corporal (IMC). Las variables relacionadas con el procedimiento fueron: lateralidad de la anastomosis (uni o bilateralmente), tiempo entre la salpingectomía (SPG) y la RAT (en meses), intervalo de tiempo entre RAT y embarazo (en meses). Las variables relacionadas con la fecundidad posquirúrgica fueron: características del embarazo (nacidos vivos, embarazos durante la investigación, abortos espontáneos, embarazos anembrionados, embarazos con implantación ectópica y óbito fetal). Para obtener estas mediciones se realizó seguimiento mensual a las pacientes.

La tabulación de los datos obtenidos, la elaboración de tablas y gráficos se realizó mediante los programas Microsoft Excel $2010^{\circledR}$ y IBM SPSS (Statistical Package for the Social Sciences) Statistics para Mac versión 22.0 ${ }^{\circledR}$. Para el análisis descriptivo de las variables cuantitativas se determinó la media, mediana, desviación estándar, valor mínimo y máximo. Posteriormente se procedió a efectuar un análisis inferencial mediante la curva de supervivencia de Kaplan Meier, para determinar el tiempo transcurrido entre la fecha de RAT y el embarazo de las pacientes. Se consideró estadísticamente significativo un valor $\mathrm{p}<0,05$. Durante este proceso se siguieron las líneas de recomendación de la Declaración de Helsinki.

\section{RESULTADOS}

Se seleccionaron un total de 344 pacientes del departamento de Ginecología y Obstetricia del hospital regional Teodoro Maldonado Carbo de la ciudad de Guayaquil que presentaron como diagnóstico infertilidad de origen tubárico (N971 según el CIE - 10). Un total de 51 pacientes 
cumplieron con los criterios de inclusión del estudio. De las 344 pacientes, el 29\% ( $n=100)$ fue candidato para la reanastomosis tubárica; y del referido porcentaje, solo el $51 \%(n=51)$ se realizó el procedimiento (Figura 1).

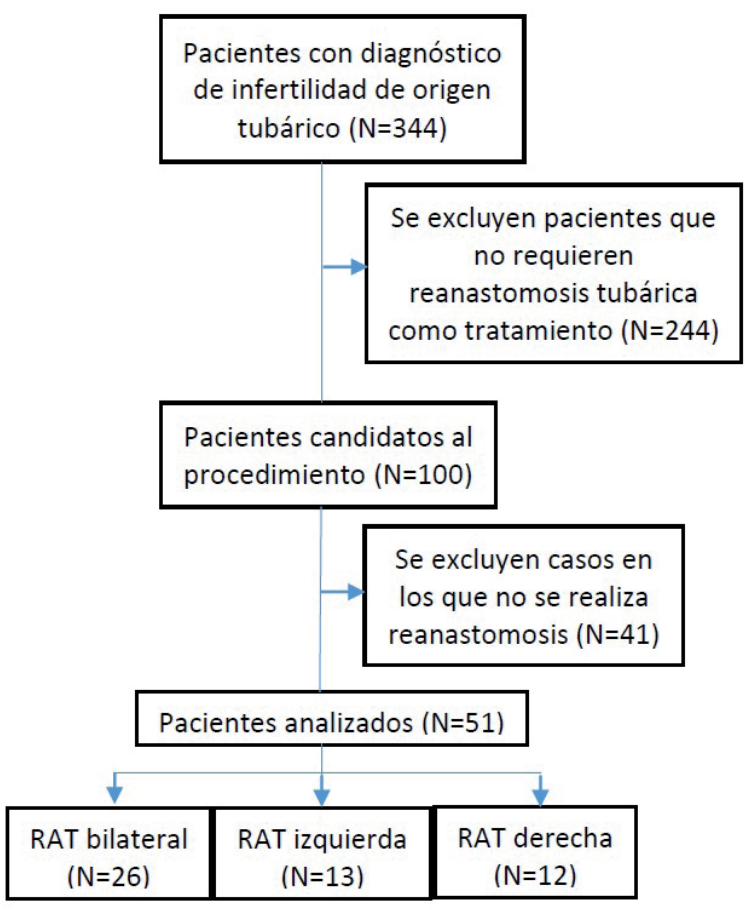

Figura 1. Flujograma de pacientes seleccionados Fuente: base de datos de HTMC

La edad media de las pacientes en el estudio fue de $34 \pm 3.6$ años, mientras que sus parejas 34.4 \pm 4.6 años. El 26\% $(n=13)$ de las mujeres desearon volver a concebir dentro de su mismo compromiso; el $66 \%(n=34)$ con una nueva pareja, ésta con o sin hijos de compromisos anteriores, y el $8 \%$ $(\mathrm{n}=4)$ solicitó RAT por el fallecimiento de uno de sus hijos. El intervalo de tiempo entre la SPG y la RAT fue de $80 \pm 47.3$ meses, (Tabla 1 y figura 2).

\section{TABLA 1. CARACTERÍSTICAS GENERALES DE LOS PACIENTES}

\begin{tabular}{ll}
\hline Antecedentes obstétricos & Mediana \\
Gestas & 3 \\
Partos & 2 \\
Cesáreas & 2 \\
Aborto & 1 \\
Hijos vivos & 1 \\
& Promedio \pm DS \\
IMC (kg/m²) & $27.12 \pm 4.5$ \\
Edad al momento de RAT & $34.0 \pm 3.6$ \\
Edad al momento de esterilización & $27.3 \pm 5.2$ \\
Periodo entre SPG y RAT (meses) & $80.0 \pm 47.3$ \\
Periodo ente RAT y embarazo (meses) & $8.4 \pm 5.6$ \\
Edad de pareja (años) & $34.4 \pm 4.6$
\end{tabular}

Fuente: base de datos de HTMC.

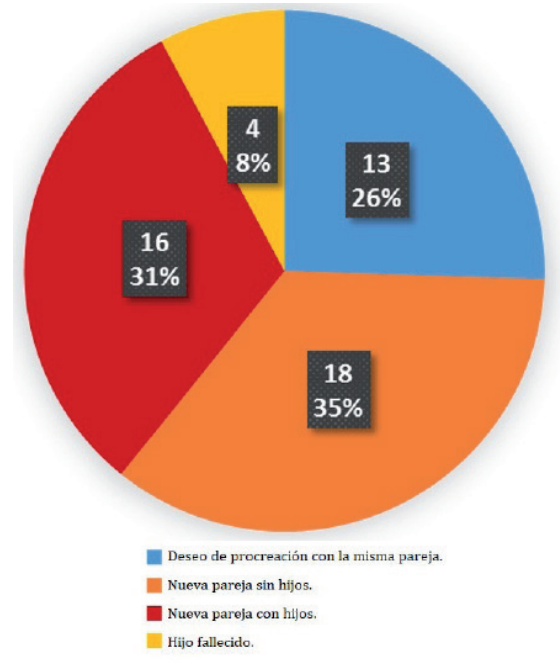

El 49\% ( $n=25)$ de las reanastomosis realizadas resultaron unilaterales. Se confirmó la cromopertubación positiva en todos los casos antes de finalizar el procedimiento.

Después de 6 semanas se solicitó a las pacientes una histerosalpingografía de control. Del total, 6 pacientes no se realizaron dicho examen, mientras que de las 45 restantes, el 20\% $(n=9)$ presentó obstrucción tubárica bilateral; el resto obtuvo prueba de Cotte positiva uni o bilateralmente. Durante el periodo analizado, se obtuvo un total de 28 gestas pertenecientes a 18 pacientes del estudio. Se reportaron 3 productos nacidos vivos por cesárea (11\%), 2 embarazos en curso durante la investigación (7\%), 13 abortos espontáneos (46\%), 2 embarazos anembrionados (7\%) y 1 óbito fetal de 35 semanas de gestación (4\%). Por último, se presentaron 7 embarazos con implantación ectópica (25\%). En el 68\% de las gestas, la recanalización fue de tipo bilateral; en los embarazos uterinos viables se les realizó anastomosis ístmico-ístmica en las 5 pacientes. Cabe recalcar que 19 de los embarazos (68\%) se reportaron dentro del grupo de edad de menores de 35 años, (Figura 3).

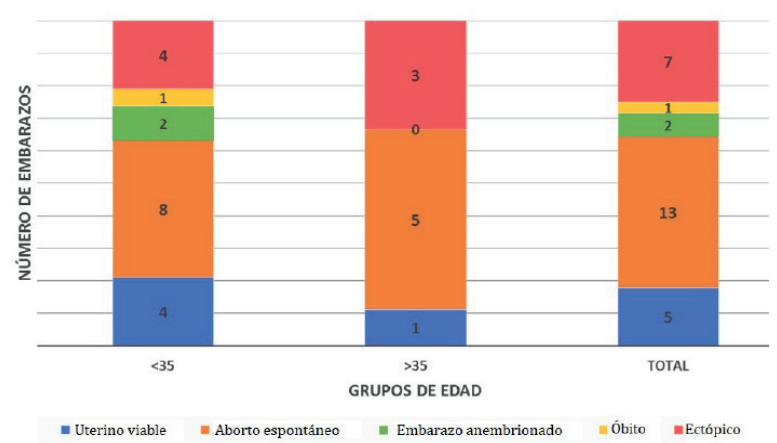

Figura 3. Tipo de gesta según grupos de edad. Fuente: base de datos de HTMC 
Se realizó la curva de Kaplan - Meier con la finalidad de determinar la probabilidad de embarazo según el tiempo transcurrido desde la RAT basándola en los datos de las mujeres gestantes (Figura 4). La curva determinó que las pacientes tienen una probabilidad en ascenso de concebir de hasta el $0.4 \%$ durante los primeros 20 meses, momento en el cual alcanza una meseta y se mantiene estable en el porcentaje mencionado.

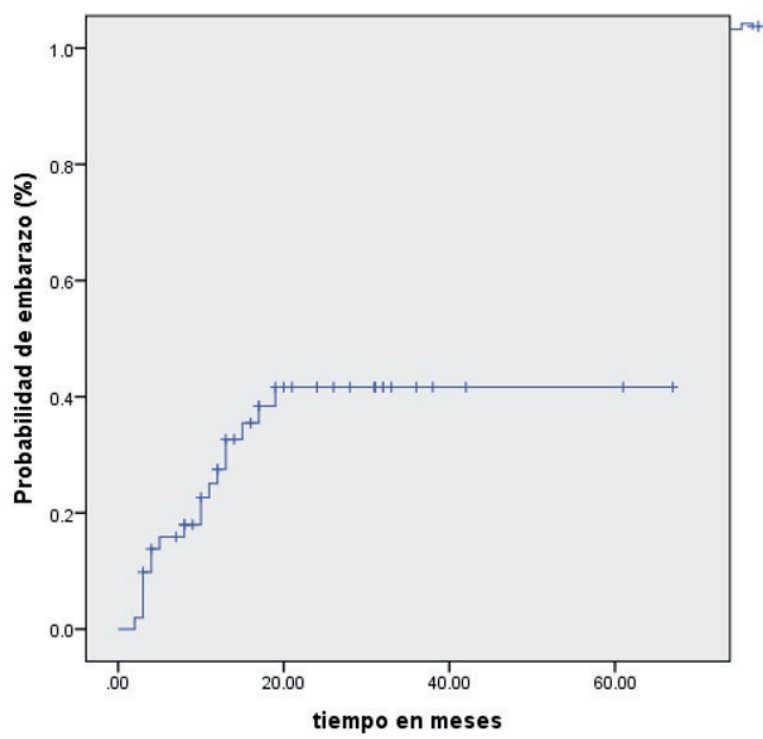

Figura 4. Curva de Kaplan Meier para determinar el porcentaje de probabilidad de embarazo a partir de la RAT.

Fuente: base de datos de HTMC.

Treinta de las 33 pacientes que no lograron concebir siguieron con los controles ginecológicos. De estas, a 3 pacientes se les recomendó TRA, a una paciente se le realizó salpingectomía bilateral por presentar hidrosalpinx en ambas trompas. Por otra parte, a 2 pacientes se les realizó salpingectomía unilateral posterior a embarazo ectópico. El intervalo de tiempo transcurrido para este grupo de pacientes es de $21.1 \pm 14.6$ meses.

Por último, se compararon los parámetros hormonales y de IMC de todas las pacientes post reanastomosis tubárica Se dividieron en dos grupos: las gestantes $(\mathrm{n}=18)$ y las no gestantes $(\mathrm{n}=33)$. Dentro de las gestantes, el 95\% presentó rangos hormonales dentro de los parámetros normales; el $75 \%$ se encontró dentro de los rangos normales de perfil lipídico y el $72 \%$ presentó glicemias e índice HOMA-IR sin alteraciones. En cuanto al índice de masa corporal se reportó un $22 \%(n=4)$ de las gestantes dentro del rango ideal de 18.5 - $24.9 ; 39 \%(n=7)$ con sobrepeso y un $33 \%$ $(\mathrm{n}=6)$ con un índice de obesidad mayor a 30.
De igual manera, el 93\% de las no gestantes mostró rangos hormonales normales, 70\% perfil lipídico normal y 83\% glicemia e índice HOMA no alterados. En cuanto al índice de masa corporal, el $39 \%(n=13)$ se encontraron dentro del rango ideal, $42 \%(n=14)$ con sobrepeso y un $18 \%(n=6)$ reportó un índice de obesidad mayor a 30. El IMC en promedio fue de $27.1 \pm 4.5$ para todas las pacientes.

\section{DISCUSIÓN}

Se revisaron los principales motivos que conllevaron a este procedimiento. En este medio se encontró que la mayoría refirió una nueva pareja sexual, seguido por deseos de procreación con la misma pareja y finalmente por el fallecimiento de un hijo. De igual manera, Schepens et al, en su investigación efectuada en Holanda en el 2011 determinaron que el deseo de procrear con una nueva pareja comprendió el $80 \%$ de los casos de RAT, el 20\% restante fue por mortalidad infantil o por deseos de concebir con la misma pareja. ${ }^{8}$

El 60\% de las mujeres que posteriormente optaron por la recanalización se encontraron dentro del grupo etario de menores de 35 años de edad. Asi mismo, Ramalingappa et al, en su estudio efectuado en India en el 2012, obtuvieron valores similares con un $84 \%$ de sus pacientes dentro de dicho grupo de edad y un promedio de intervalo de tiempo entre intervenciones de 63 meses ó 5.25 años. ${ }^{10}$ Llama la atención la edad temprana en la que mujeres de diferente entorno socioeconómico recurren a la esterilización.

En el 2013, Ray-Gaheer et al, en el Reino Unido, consiguieron un total de 26 embarazos de 48 pacientes. El 59.5\% de las gestas ocurrió en RAT bilateral. ${ }^{11}$ En este estudio, solo un embarazo intrauterino viable ocurrió en una recanalización derecha, por lo que la probabilidad de concepción y progreso del embarazo favorece a la bilateral.

El promedio de tiempo transcurrido entre la RAT y el embarazo fue de $8.4 \pm 5.6$ meses, un intervalo más corto que los $9.5 \pm 2.3$ meses evidenciado por Ray-Gaheer et al, ${ }^{11}$ y los 13.6 meses descrito por Tan H y Loh S mediante laparotomía en el 2010, en Singapur. ${ }^{7}$ Estos promedios similares de periodo transcurrido entre RAT y embarazo exponen la eficacia del procedimiento.

La curva de Kaplan Meier demostró que las pacientes tienen una probabilidad en ascenso de concebir de hasta $40 \%$ durante los primeros 
20 meses, hasta llegar a una meseta en el valor mencionado. Schepenas et al, registraron una probabilidad de hasta $0.6 \%$ durante los primeros 40 meses, punto en el que alcanza su meseta. ${ }^{8}$ De acuerdo a los resultados de la curva, la tasa de gestas en las reanastomosis realizadas en el HTMC guardan relación con las descritas en otros estudios, reluciendo la efectividad de la intervención a nivel mundial.

Sook H et al, reportaron en el 2012 en Korea, que el $85.1 \%$ de 886 pacientes lograron concebir, de los cuales $92.3 \%$ resultaron en productos nacidos vivos, y el restante fueron abortos espontáneos y embarazos ectópicos. ${ }^{12}$ Monteith C et al, en el 2014 en EEUU reportaron 31 embarazos de 70 pacientes, de los cuales el $64.5 \%$ se confirmaron como productos nacidos vivos, $25.8 \%$ pérdidas y $6.45 \%$ gestas en curso. ${ }^{13}$ Resalta la alta incidencia de aborto espontáneo y embarazo ectópico en este estudio, que contradice los valores alcanzados alrededor del mundo.

Por último, los antecedentes antropométricos y valores hormonales, lipídicos, y glicémicos también fueron considerados como posibles factores influyentes en un embarazo posrecanalización tubárica. Tanto las gestantes como las no gestantes presentaron casi en su totalidad (94.4\%) valores hormonales normales de acuerdo a los rangos utilizados por el HTMC. En referencia al perfil lipídico y glicémico, el porcentaje de pacientes con valores normales disminuyó ligeramente. Por lo tanto, dentro del presente estudio, el carácter hormonal no tuvo un impacto significativo sobre la probabilidad de embarazo. Sin embargo, la evidencia sobre el índice de masa corporal resalta en comparación a otras investigaciones.

Resulta discordante la incidencia de gestas en pacientes con IMC elevados, que no se esperaban en el pronóstico inicial. Por ende, es menester una investigación endocrinológica exhaustiva cuyo propósito incluya el bienestar integral de la mujer.

En varios estudios como el de Jayakrishnan ${ }^{14}$ y Ayoubi J, ${ }^{15}$ la técnica, la localización de SPG y la longitud de la trompa, juegan un papel esencial en el éxito de la reanastomosis. De la misma manera, el hombre también debe ser investigado como posible causa de infertilidad en una pareja; sin embargo, solo el $37.3 \%$ de los espermatogramas fueron reportados y en total,
19.6\% confirmaron alteraciones como hipospermia y astenospermia. ${ }^{16}$

\section{CONCLUSIONES Y RECOMENDACIONES}

La reanastomosis tubárica en el presente estudio alcanzó una tasa de embarazos acumulados de $55 \%$, con el $35 \%$ de las pacientes concibiendo. Sin embargo no garantiza la viabilidad de un embarazo. Una correcta anastomosis no es el único factor determinante para que una mujer logre concebir; se debe tomar en cuenta la edad en la que se realiza el procedimiento, preferiblemente menor de 35 años; el estado integral de la mujer y de su pareja.

Otro factor que se debe considerar es el método de esterilización al que se sometió previamente la paciente. Dependiendo de la técnica y las complicaciones durante este primer procedimiento, afectará la probabilidad de obtener un embarazo viable posterior a la reanastomosis tubárica. Es por esto que se recomienda, dentro de lo posible, que los médicos responsables de la SPG describan detalladamente el procedimiento y hallazgos quirúrgicos, para así orientar, posteriormente, al médico especialista en infertilidad sobre el tratamiento más adecuado para su paciente.

Las mujeres que deseen revertir la esterilización tubárica deben ser conscientes de las posibilidades de fracaso, por lo que es indispensable una investigación y asesoramiento pertinente para descartar cualquier factor de mal pronóstico.

En casos de que la cirugía reproductiva no sea favorable, se puede plantear la terapia de reproducción asistida para lograr la concepción. La pareja y el médico deben mantener una comunicación abierta y sincera para decidir correctamente qué tratamiento brindará el mayor beneficio para los involucrados. ${ }^{17}$

REFERENCIAS BIBLIOGRÁFICAS

1. Tanaka Y, Tajima H, Sakuraba S, Shimokawa R, Kamei K. Renaissance of Surgical Recanalization for Proximal Fallopian Tubal Occlusion: Falloposcopic Tuboplasty as a Promising Therapeutic Option in Tubal Infertility. J Minim Invasive Gynecol. 2011 Sep-Oct;18(5):651-9.

2. American Society for Reproductive Medicine. Role of tubal surgery in the era of assited reproductive technology: a committee opinion. Fertil Steril. 2015 Jun;103(6):e37-43.

3. Grange J, Kruger T.F, Steyn D.W, et. al. Fallopian Tuba Reanastomosis by Laparotomy versus Lapa- 
roscopy: A Meta-Analysis. Gynecol Obstet Invest. 2012;74(1):28-34.

4. UN. Patrón de uso de Anticonceptivos a nivel Mundial. Organización de las Naciones Unidas, Departamento de Asuntos Economicos y Sociales, División de población. Año 2013. 2p.

5. Noreda L. El futuro de la cirugía reproductiva. Rev. peru. ginecol. obstet., Lima, v.58, n.3, 2012

6. Van de Water M, Bosteels J, De Sutter P. Laparoscopic non micro-surgical tubal reanastomosis: A retrospective cohort study. Eur J Contracept Reprod Health Care. 2015 Jun;20(3):193-200.

7. Tan H, Loh S, Microsurgical Reversal of Sterilisation - Is This Still Clinically Relevant Today?. Ann Acad Med Singapore. 2010 Jan;39(1):22-6.

8. Schepens J, Mol B, Wiegerinck M, et.al. Pregnancy outcomes and prognostic factors from tubal sterilization reversal by sutureless laparoscopical re-anastomosis: a retrospective cohort study. Hum Reprod. 2011 Feb;26(2):354-9.

9. Coughlan C, Li TC. Surgical Management of tubal disease and infertility. Obstetrics, Gynaecology and Reproductive Medicine, abril 2009, Volume 19 , Issue 4, 98 - 105.

10. Ramalingappa A, Yashoda. A Study on Tubal Recanalization. J Obstet Gynaecol India. 2012 Apr; 62(2): 179-183.
11. Ray-Gaheer N, Pandravada A, Kumar R. Fertility outcome following reversal of sterilisation by open laparotomy: five years retrospective study. In: BenRafael Z, editor. 17th World Congress on Controversies in Obstetrics, Gynecology e Infertility (COGI)

12. Sook H, Sun B, Sik G. High pregnancy rate after microsurgical tubal reanasotomosis by temporary loose parallel 4-quadrant sutures technique: a long term follow-up report on 961 cases. Hum Reprod. 2012 Jun;27(6):1657-62.

13. Monteith C, Berger G, Zerden M. Pregnancy Succes After Hysteroscopic Sterilization Reversal. Obstet Gynecol. 2014 Dec;124(6):1183-9.

14. Jayakrishnan K, Baheti S. Laparoscopic tubal sterilization reversal and fertility outcomes. J Hum Reprod Sci. 2011 Sep;4(3):125-9.

15. Ayoubi J, De la Jolilniere J, Feki A, et. al. Laparoscopic Tubal Anastomosis. An Assessment of the “One-Stitch Technique” Reprod Sys Sexual Disorders 2013 2:119.

16. Allahabadia G, Merchant R. Fallopian tube recanalization: lessons learnt and future challenges. Womens Health (Lond Engl). 2010 Jul;6(4):531-48, quiz 548-9.

17. Tanaka Y, Tajima H. Falloposcopic tuboplasty as an option for tubal infertility: an alternative to in vitro fertilization. Fertil Steril. 2011 Jan;95(1):441-3. 